\title{
OPEN Associations of the oral microbiota and Candida with taste, smell, appetite and undernutrition in older adults
}

\author{
Kristina S. Fluitman ${ }^{1,2,3}$, Tim J. van den Broek ${ }^{4}$, Max Nieuwdorp ${ }^{1,5}$, Marjolein Visser ${ }^{3,6}$, \\ Richard G. IJzerman ${ }^{1} \&$ Bart J. F. Keijser ${ }^{4,7 凶}$
}

Poor taste and smell function are widely thought to contribute to the development of poor appetite and undernutrition in older adults. It has been hypothesized that the oral microbiota play a role as well, but evidence is scarce. In a cross-sectional cohort of $\mathbf{3 5 6}$ older adults, we performed taste and smell tests, collected anthropometric measurements and tongue swabs for analysis of microbial composition (16S rRNA sequencing) and Candida albicans abundance (qPCR). Older age, edentation, poor smell and poor appetite were associated with lower alpha diversity and explained a significant amount of beta diversity. Moreover, a lower Streptococcus salivarius abundance was associated with poor smell identification score, whereas high $C$. albicans abundance seemed to be associated with poor smell discrimination score. In our population, neither the tongue microbiota, nor $C$. albicans were associated with poor taste or directly with undernutrition. Our findings do suggest a host-microbe interaction with regard to smell perception and appetite.

Older adults over 65 years old make up the fastest growing age group worldwide. Currently, they comprise $20 \%$ of the European population ${ }^{1}$. These older adults are particularly prone to the development of undernutrition, which affects $9 \%$ of the community-dwelling and $28 \%$ of the institutionalized older adults ${ }^{2}$. Undernutrition is associated with various adverse events ${ }^{3,4}$ and is predominantly caused by a phenomenon coined "Anorexia of Aging" . This is the reduction in appetite and energy intake that is often observed at older age. Factors such as cognitive impairments, depression, and polypharmacy, as well as poor taste and smell, poor dentition, and poor oral health are all considered to contribute to Anorexia of Aging ${ }^{6,7}$. It can be hypothesized that the oral microbiota is a new important factor in the Anorexia of Aging phenomenon, either by influencing taste or smell function ${ }^{8-11}$, or by other-as of yet unknown-mechanisms.

The oral microbiota constitute a complex, highly diverse microbiological community in the oral cavity ${ }^{12}$. Due to the anatomical proximity of the oral microbiota to taste and smell receptors, the two entities are thought to influence each other in several ways ${ }^{13}$. First, the oral microbiota metabolize and synthesize sensory active substances, affecting taste and smell receptor signaling in a process called "sensory adaption" 13 . Second, the oral microbiota may interact with the oral microenvironment, which in turn affects taste and smell function ${ }^{9}$. Finally, it was shown that germfree mice (lacking oral bacteria) developed altered olfactory epithelium compared to conventional mice and had a different olfactory neuronal response to olfactory stimuli ${ }^{14}$. Some studies have evaluated the association of the oral microbiota with taste function in humans. However, these studies reported inconsistent results, were limited in sample size, and did not focus specifically on older adults ${ }^{8-11}$. To our knowledge, we are the first to study the association of the oral microbiota with taste, smell, appetite and undernutrition in older adults.

\footnotetext{
${ }^{1}$ Department of Internal Medicine, Amsterdam University Medical Center, Location VUmc, Amsterdam, The Netherlands. ${ }^{2}$ Wallenburg Laboratory, Department of Molecular and Clinical Medicine, Sahlgrenska Academy, University of Gothenburg, Gothenburg, Sweden. ${ }^{3}$ Amsterdam Public Health Research Institute, Amsterdam, The Netherlands. "Department of Microbiology and Systems Biology, TNO Healthy Living, Zeist, The Netherlands. ${ }^{5}$ Department of Vascular Medicine, Amsterdam University Medical Center, Location AMC, Amsterdam, The Netherlands. ${ }^{6}$ Department of Health Sciences, Faculty of Science, Vrije Universiteit Amsterdam, Amsterdam, The Netherlands. ${ }^{7}$ Department of Preventive Dentistry, Academic Center for Dentistry Amsterdam, University of Amsterdam and Vrije Universiteit Amsterdam, Amsterdam, The Netherlands. ${ }^{\varpi}$ email: Bart.keijser@ tno.nl
} 
In addition to the oral microbiota, the oral mycobiota-particularly Candida-may be associated with undernutrition. In a study of 218 young adults, candidiasis patients and healthy carriers of Candida albicans had significantly higher taste thresholds and were more likely to suffer from taste disorders than non-carriers. Oral candidiasis was also found to be associated with undernutrition ${ }^{7}$ and lower energy and protein intake ${ }^{15}$ in hospitalized older adults. Preliminary observational data suggested that the taste disorders ${ }^{16}$ and energy intake ${ }^{15}$ improved after treatment with antimycotica. Whether oral C. albicans is associated with poor smell, poor appetite or nutritional status at sub-pathological abundances is not yet known.

In this study, we aim to evaluate the oral microbiota and explore C. albicans from the tongue dorsum of 356 Dutch community-dwelling older adults in relation to taste and smell function, as well as poor appetite and undernutrition. A deeper understanding of the oral microbiota in relation to taste, smell, and appetite may further illuminate the complex pathophysiology of undernutrition in older adults and could possibly allow for the identification of preventative or therapeutic targets.

\section{Results}

Participant characteristics. A total of 356 well-phenotyped Dutch community-dwelling older adults from the ongoing Longitudinal Aging Study Amsterdam (LASA) ${ }^{17-19}$ were included in this cross-sectional cohort study. On average, participants were 73 years old (ranging from 65-93) and 207 (58.1\%) were male. Undernutrition occurred in $76(21.3 \%)$ participants, either based on low BMI $\left(<20 \mathrm{~kg} / \mathrm{m}^{2}\right.$ if $<70$ years, or $<22 \mathrm{~kg} / \mathrm{m}^{2}$ if $\geq 70$ years $)^{20}(n=39)$ or on $>5 \%$ body weight loss averaged over the last 2 years $(n=43)$, or both. Poor appetite occurred in $20(5.6 \%)$ participants based on a Council of Nutrition Appetite Questionnaire (CNAQ) score $<28^{21}$. Measured poor smell occurred in $16.5 \%, 22.0 \%, 19.7 \%$, and $18.0 \%$ of participants (poor total smell score, poor smell threshold score, poor smell discrimination score, and poor smell identification score, respectively). Poor sour, salty, and umami taste scores were observed most often $(31.5 \%, 37.7 \%$, and $32.4 \%$, respectively), whereas poor Bitter, sweet, and total taste scores were less common (7.3\%, 15.5\%, and 9.3\%, respectively). An overview of all participant characteristics is shown in Supplementary Table S1.

Associations of the tongue microbiota with taste, smell, appetite, and undernutrition. Microbiota composition was assessed by $16 \mathrm{~S}$ rRNA sequencing of tongue swabs. Median sequencing depth was 94,703 reads/sample. In our cohort, 5367 amplicon sequencing variants (ASVs) were identified. Of these ASVs, 4429 were matched to known bacterial species from the Human Oral Microbiome Database (HOMD) with a sequence identity match $>90 \%{ }^{22}$. The other 938 ASVs were minority members with a mean relative abundance of $0.000126 \% \pm 0.001167$. When filtering the $16 \mathrm{~S}$ data to include only those ASVs contributing to the first $97.5 \%$ of all count data, 386 ASVs with an abundance relative to all count data of $\geq 0.01 \%$ each remained. These ASVs were matched to the HOMD with a mean $99.5 \%$ sequence identity match. The abundance filter was applied prior to all bio-statistical analysis, except for those concerning alpha-diversity. Sequencing of ten samples yielded less than 30.000 raw sequence reads and were excluded from further analysis.

First, the overall microbial composition was evaluated using alpha- and beta-diversity measures.

Alpha-diversity is an ecological measure used to indicate the intra-individual microbial diversity (i.e. how diverse is a participant's oral microbiota). It was calculated using Shannon and inverse Simpson indices. Betadiversity is used to indicate inter-individual dissimilarity in microbiota composition (i.e. how much does the overall microbiota composition differ between participants). It was calculated using the Bray-Curtis dissimilarity measure. We explored the univariate associations of all measured clinical variables with alpha- and beta-diversity (Fig. 1). The heatmap in Fig. 1 depicts the extent to which the clinical variables were associated with Shannon and Inverse Simpson alpha-diversity indices, analyzed with linear regression. Poor smell identification was significantly associated with lower diversity ( $p=0.009$ for Inverse Simpson), but none of the other smell or taste scores were. Poor appetite was also associated with lower alpha-diversity ( $p<0.001$ for Shannon and Inverse Simpson), but undernutrition, bodyweight, BMI, weight change, Fat Free Mass Index (FFMI), and Appendicular Skeletal Muscle Mass (ASMM) were not. Of the remaining variables, alcohol use, higher Centre for Epidemiologic Studies Depression (CESD)-score (indicating less depressive symptoms), and male sex were significantly associated with higher alpha-diversity. Having only some or no teeth, older age, more medication use, low or moderate education, xerostomia, and smoking were all significantly associated with lower alpha-diversity. The bar graphs in Fig. 1 depict the extent to which the clinical variables explained the variance in Bray-Curtis dissimilarity, analyzed with PERMANOVA analysis. Of all clinical variables, dentition explained the most variance in tongue microbial composition $\left(\mathrm{R}^{2}=0.10, p<0.001\right)$, followed by age $\left(\mathrm{R}^{2}=0.08, p<0.001\right)$. Of the smell scores, again only poor smell identification score explained a significant amount of variance $\left(\mathrm{R}^{2}=0.02, p=0.005\right)$. So did poor appetite $\left(R^{2}=0.02, p=0.003\right)$, but none of the taste scores did, nor did undernutrition, weight, weight change, BMI, FFMI, or ASMM. Of the other variables, carbohydrate intake, medication use, education, xerostomia, and smoking explained significant amounts of variance. The precise coefficients of the analyses depicted in Fig. 1 are reported in Supplementary Table S2.

The Bray-Curtis dissimilarity is further visualized in Fig. 2, depicting a multi-dimensional scaling plot, in which each point represents the microbial composition of an individual participant. The further two points are apart, the more they differ based on Bray-Curtis dissimilarity. Samples are colored based on appetite, smell identification score, total taste score, or undernutrition. As was shown in Fig. 1, Bray Curtis dissimilarity is greater between participants with and without poor appetite or poor smell than between participants with and without poor taste or undernutrition. Next, we performed canonical correspondence analysis (CCA). This is a multivariate statistics technique in which correlations between two datasets are analyzed. Here, poor total taste score and poor smell identification score, or poor appetite and undernutrition make up one dataset, whereas the tongue microbiota (i.e. the abundance of all individual ASVs) make up the other. Again both poor appetite $(p=0.001)$ 


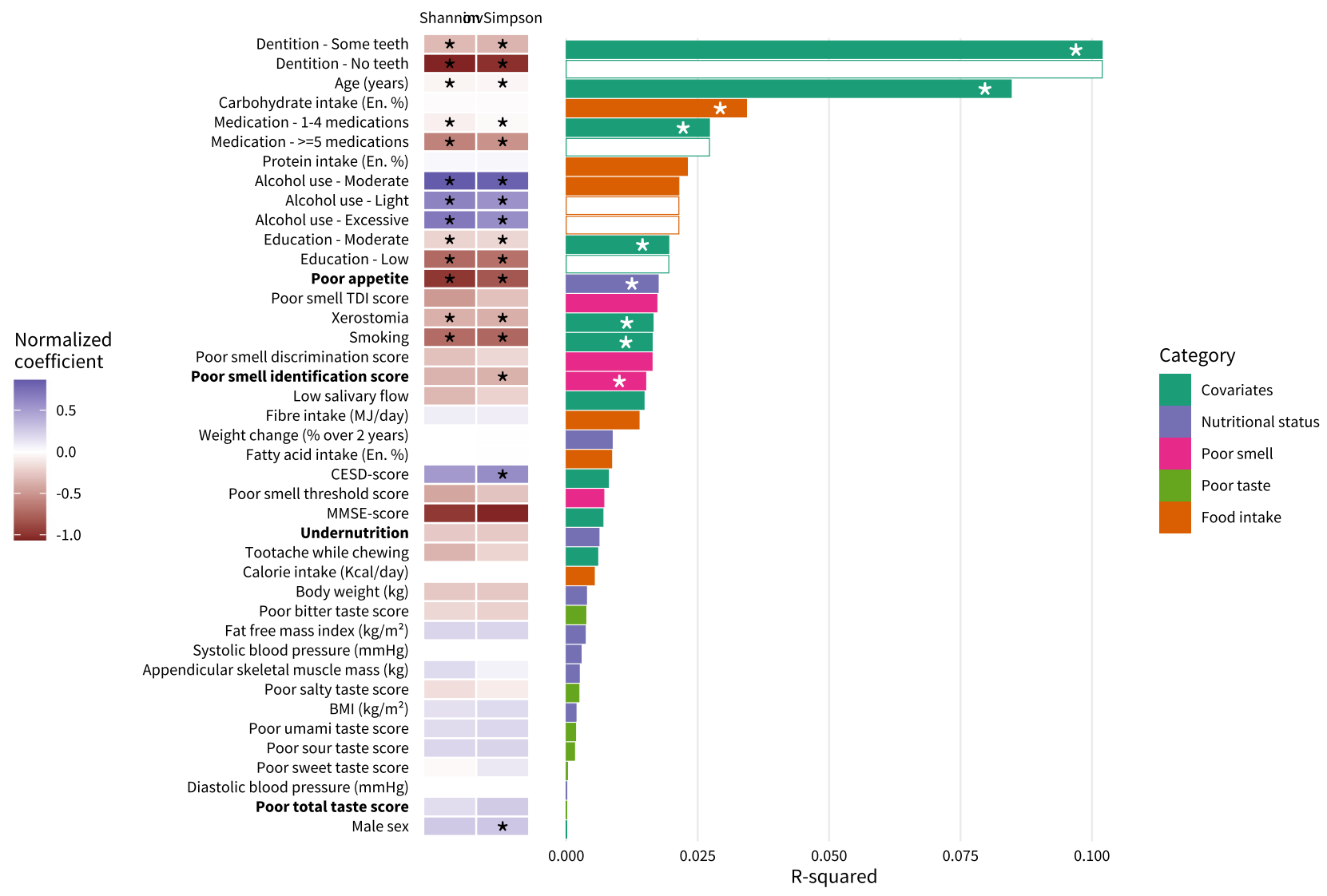

Figure 1. Association of clinical variables with microbiota alpha-diversity and beta-diversity. The heatmap depicts the normalized linear regression coefficients for the associations of clinical variables with Shannon and Inverse Simpson alpha-diversity. Blue indicates a positive association and red a negative association. Bar graphs depict the amount of variance explained by each clinical variable in the Bray-Curtis beta-diversity measure based on PERMANOVA models. Bars are colored based on the type of variable. White bars are shown for values of the same categorical variable. The variables poor appetite, poor smell identification, undernutrition, and poor total taste score concern our main hypotheses and are in written bold text. Asterisks indicate statistical significance at a $p$ value $<0.01$. TDI score Threshold discrimination identification score, CESD Centre of epidemiological studies depression scale, MMSE Mini-mental state exam, BMI Body mass index.

and poor smell identification $(p=0.010)$ were significantly correlated with the microbiota data, whereas poor taste and undernutrition were not. This is depicted in Fig. 3, as well as the bacterial species driving these correlations. Detailed results of the CCA analyses can be found in Supplementary Table S3.

Finally, we examined the associations of individual microbial taxa with poor smell, taste, appetite, and undernutrition using DESeq analyses. In our univariate analyses, we identified various taxa that were differentially abundant for our clinical outcomes. These results are depicted in Supplementary Fig. 1 and Supplementary Table S4. Because our previous analyses (Fig. 1) identified age and dentition as most important microbiota determinants, we adjusted our DESeq analyses for both age and dentition (Fig. 4 and Supplementary Table S5). Then only the association of a lower abundance of Streptococcus salivarius with poor smell identification score remained $(\log 2$ Fold Change $=-0.69$, adjusted $p=0.003)$. No other taxa were significantly differentially abundant for any of the other taste or smell scores, nor for appetite or undernutrition. Altogether, we demonstrated that both poor appetite and poor smell function are univariately associated with the overall tongue microbiota composition (alpha- and beta-diversity) in older adults. We also showed that participants with poor smell identification have a lower abundance of $S$. salivarius after adjustment for age and dentition.

Associations of Candida albicans with taste, smell, appetite, and undernutrition. The abundance of $C$. albicans was determined with qPCR of the tongue swabs. A cross-table with the proportions of participant with no C. albicans, low C. albicans abundance, and high C. albicans abundance that have poor taste, smell, appetite or undernutrition can be found in Supplementary Table S6. With logistic regression analyses (both crude and adjusted), we found C. albicans carriers (low or high abundance versus no C. albicans present) did not have a higher risk of poor taste, poor appetite, or undernutrition (Table 1). However, in our population, participants with high abundance of C. albicans did have 4.7 times higher odds for poor smell discrimination than participants with no C. albicans, after adjustment for age, dentition, sex, educational status, smoking status, medication use, CESD-score, MMSE-score (Bonferroni adjusted $p$ value: 0.048). 


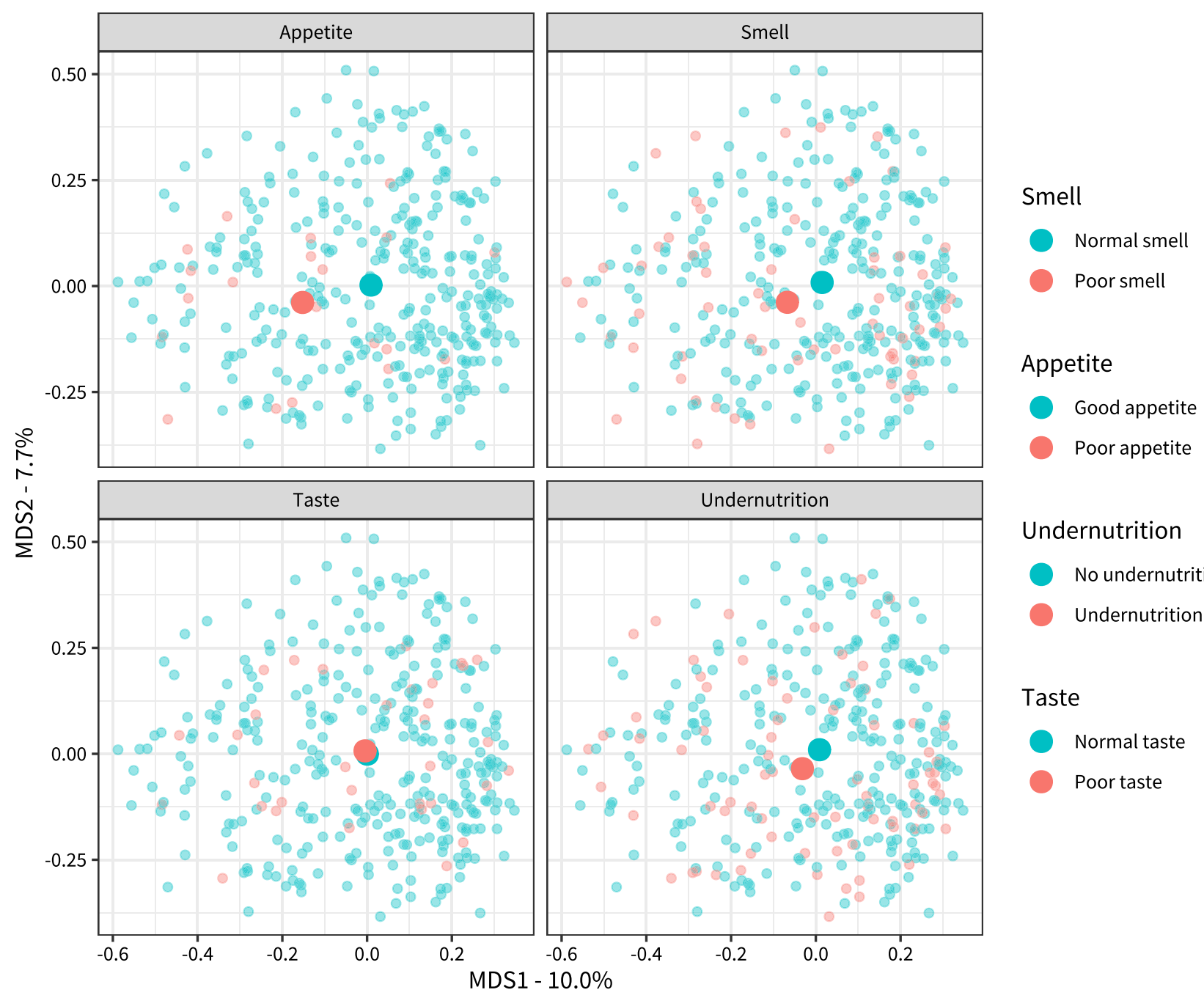

Figure 2. Ordination plots for Bray-Curtis dissimilarity colored for poor taste, smell, appetite, and undernutrition. Multi-dimensional scaling (or principal coordinate analysis) of the Bray-Curtis betadissimilarity in microbiota data. Each dot represents the microbiota sample of a single participant. Distance between samples represents the dissimilarity of these samples from each other. The axes represent $10.0 \%$ and $7.65 \%$ of variation in Bray-Curtis Distance. Samples are colored according to appetite, smell, taste, and undernutrition. MDS Multi-dimensional scaling.

\section{Discussion}

In this study, we aimed to evaluate the tongue microbiota and explore C. albicans in relation to poor appetite and undernutrition in older adults. In our sample of Dutch community-dwelling older adults, alpha- and betadiversity of the tongue microbiota were associated with poor smell and poor appetite, but not with poor taste or undernutrition. Moreover, a lower abundance of $S$. salivarius was significantly associated with poor smell identification and a high abundance of $C$. albicans seemed to be associated with poor smell discrimination.

To our knowledge, we are the first to study the associations of the oral microbiota with olfactory function in humans. Lower intra-individual (alpha) diversity was associated with poor smell identification, which also explained a significant amount of variance in inter-individual (beta) diversity, measured by Bray-Curtis dissimilarity. CCA analysis confirmed the association of oral microbiota with poor smell identification. Moreover, when studying the associations with individual bacterial taxa, a lower abundance of $S$. salivarius was associated with poor smell identification, even after adjustment for both age and dentition. S. salivarius is a prominent member of the tongue microbiota. It is a facultative anaerobic bacterium and is considered to be one of the first colonizers of the human oral cavity at birth ${ }^{23}$. Both in our study population and in a previous study of older adults, it was the single most abundant taxon on the tongue dorsum ${ }^{24}$. S. salivarius has been extensively studied for its anti-inflammatory properties and possible application as oral probiotic ${ }^{25}$. Live $S$. salivarius were shown to interfere with virulent respiratory pathogens such as Streptococcus mutans, Streptococcus sobrinus, and especially Streptococcus pyogenes which is typically involved in causing pharyngitis ${ }^{23,26,27}$. Possibly, a lower abundance of $S$. salivarius increases the risk of nasopharyngeal infections and inflammation, contributing to inflammationassociated olfactory dysfunction ${ }^{28}$. Our results suggest $S$. salivarius-based probiotic therapy may benefit older adults with hyposmia, although our findings need to be replicated and causality must be established first. 


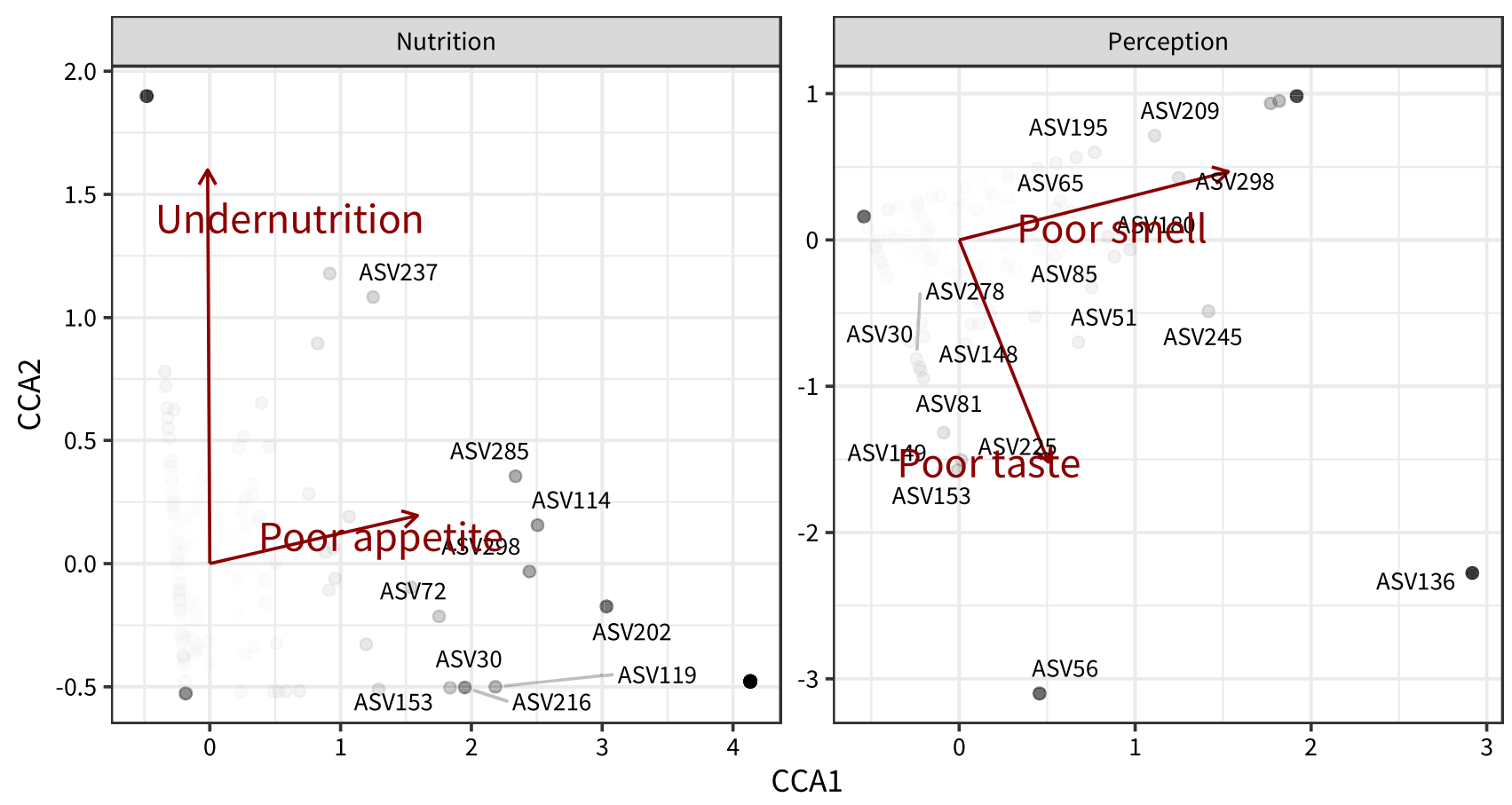

Figure 3. Canonical correspondence analysis of microbiota with poor taste, smell, appetite, and undernutrition. Canonical correspondence analysis depicting the species that most drive the correlations of the microbiota with poor appetite and undernutrition, or poor taste and poor smell. Poor appetite and poor smell identification are significantly associated with the microbiota data (CCA $p=0.010$ and $p=0.001$, respectively). Species are incorporated in the plot according to the interquartile rule, i.e. if their distance from the null-point $(0,0)$ is greater than (1.5.IQR) + Q3 (in which IQR = Interquartile Range and Q3 = the third quartile). Species names corresponding to ASV numbers can be found in Supplementary Table S3. CCA Canonical correspondence analysis, $A S V$ Amplicon sequence variance.

\section{Significant (adj. $P<0.05) \log 2 \mathrm{FC}$}

Veillonella parvula-ASV18

Veillonella dispar-ASV76

Streptococcus salivarius-ASV1

Streptococcus mitis-ASV9

Solobacterium moorei-ASV88

Selenomonas sp. HMT 478-ASV34

Prevotella salivae-ASV20

Mogibacterium diversum-ASV83

Megasphaera micronuciformis-ASV21

Lachnoanaerobaculum orale-ASV33

Haemophilus parainfluenzae-ASV6

Granulicatella adiacens-ASV23

Gemella haemolysans-ASV16

Campylobacter concisus-ASV29

Alloprevotella sp. HMT 308-ASV52

Actinomyces sp. HMT 180-ASV12

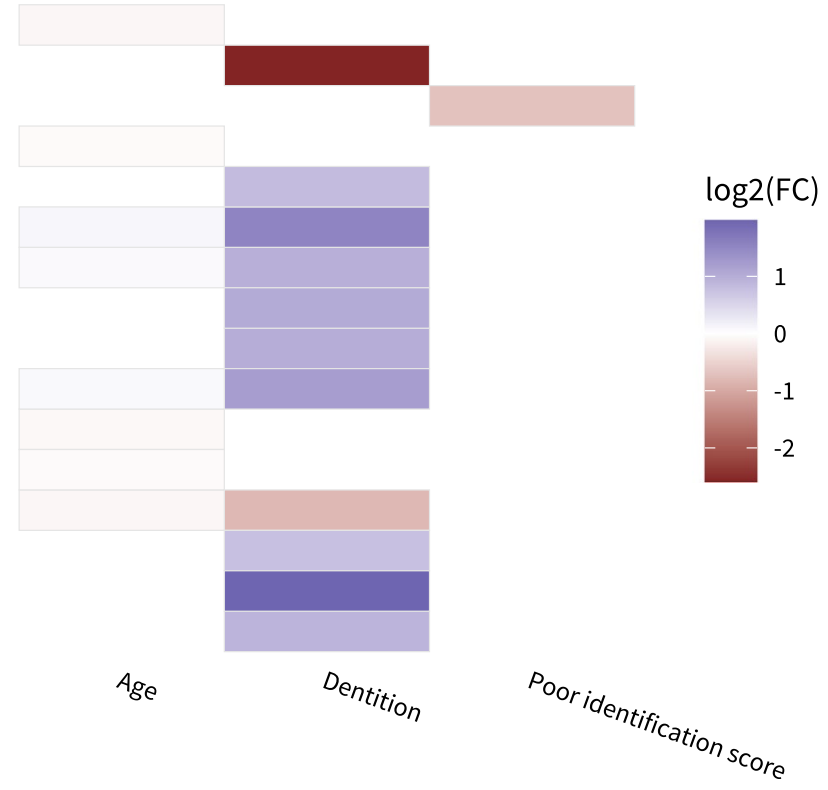

Figure 4. Microbiota taxa multivariately associated with age, dentition, poor taste, smell, appetite, and undernutrition outcomes. Heatmap depicting $\log 2$ fold change for taxa that are significantly (BenjaminiHochberg adjusted $p$ value $<0.05$ ) associated with age, dentition, all poor smell scores, all poor taste scores, poor appetite, and undernutrition based on DESeq models. The association with age is adjusted for dentition, the association with dentition is adjusted for age, and the associations with all poor taste scores, all poor smell scores, poor appetite and undernutrition are adjusted for both age and dentition. Blue indicates taxa that increase in abundance with these conditions and red indicates taxa that decrease in abundance. 


\begin{tabular}{|c|c|c|c|c|}
\hline & \multicolumn{2}{|c|}{ Low versus no C. albicans } & \multicolumn{2}{|c|}{ High versus no $C$. albicans } \\
\hline & OR (95\%-CI) & $p$ value & OR (95\%-CI) & $p$ value \\
\hline \multicolumn{5}{|c|}{ Poor total taste } \\
\hline Crude & $1.02(0.40-2.58)$ & 0.965 & $1.52(0.66-3.51)$ & 0.327 \\
\hline Adjusted & $1.01(0.39-2.66)$ & 0.972 & $1.45(0.56-3.74)$ & 0.447 \\
\hline \multicolumn{5}{|c|}{ Poor sweet taste } \\
\hline Crude & $0.74(0.37-1.47)$ & 0.384 & $0.87(0.43-1.79)$ & 0.713 \\
\hline Adjusted & $0.73(0.35-1.49)$ & 0.384 & $0.92(0.40-2.07)$ & 0.831 \\
\hline \multicolumn{5}{|c|}{ Poor salty taste } \\
\hline Crude & $0.81(0.47-1.38)$ & 0.430 & $0.63(0.37-1.06)$ & 0.084 \\
\hline Adjusted & $0.85(0.49-1.48)$ & 0.562 & $0.66(0.36-1.22)$ & 0.187 \\
\hline \multicolumn{5}{|c|}{ Poor sour taste } \\
\hline Crude & $0.99(0.57-1.72)$ & 0.962 & $0.99(0.57-1.72)$ & 0.962 \\
\hline Adjusted & $1.00(0.56-1.76)$ & 0.987 & $1.19(0.63-2.26)$ & 0.591 \\
\hline \multicolumn{5}{|c|}{ Poor bitter taste } \\
\hline Crude & $0.85(0.31-2.35)$ & 0.752 & $0.62(0.24-1.58)$ & 0.316 \\
\hline Adjusted & $0.81(0.28-2.36)$ & 0.701 & $1.10(0.35-3.39)$ & 0.875 \\
\hline \multicolumn{5}{|c|}{ Poor umami taste } \\
\hline Crude & $1.07(0.61-1.87)$ & 0.813 & $0.86(0.50-1.48)$ & 0.581 \\
\hline Adjusted & $1.12(0.62-2.03)$ & 0.708 & $1.25(0.66-2.39)$ & 0.495 \\
\hline \multicolumn{5}{|c|}{ Poor smell TDI } \\
\hline Crude & $0.85(0.24-3.01)$ & 0.804 & $1.00(0.37-2.69)$ & 0.996 \\
\hline Adjusted & $0.79(0.18-3.47)$ & 0.755 & $0.55(0.16-1.93)$ & 0.349 \\
\hline \multicolumn{5}{|c|}{ Poor smell threshold } \\
\hline Crude & $0.92(0.33-2.57)$ & 0.868 & $0.49(0.19-1.26)$ & 0.138 \\
\hline Adjusted & $0.55(0.16-1.89)$ & 0.342 & $0.28(0.09-0.92)$ & 0.036 \\
\hline \multicolumn{5}{|c|}{ Poor smell discrimination } \\
\hline Crude & $2.59(0.68-9.83)$ & 0.162 & $5.86(2.00-17.20)$ & $0.001^{\star}$ \\
\hline Adjusted & $2.45(0.58-10.40)$ & 0.225 & $4.70(1.40-15.75)$ & $0.012^{\star}$ \\
\hline \multicolumn{5}{|c|}{ Poor smell identification } \\
\hline Crude & $1.66(0.85-3.26)$ & 0.141 & $2.19(1.15-4.17)$ & 0.018 \\
\hline Adjusted & $1.67(0.83-3.37)$ & 0.152 & $1.44(0.67-3.06)$ & 0.349 \\
\hline \multicolumn{5}{|c|}{ Poor appetite } \\
\hline Crude & $0.20(0.03-1.59)$ & 0.129 & $1.77(0.68-4.58)$ & 0.239 \\
\hline Adjusted & $0.21(0.03-1.82)$ & 0.157 & $0.63(0.15-2.67)$ & 0.527 \\
\hline \multicolumn{5}{|c|}{ Undernutrition } \\
\hline Crude & $0.85(0.44-1.65)$ & 0.631 & $1.31(0.71-2.40)$ & 0.385 \\
\hline Adjusted & $0.85(0.42-1.72)$ & 0.660 & $1.09(0.52-2.27)$ & \begin{tabular}{|l|l|}
0.828 \\
\end{tabular} \\
\hline
\end{tabular}

Table 1. Logistic regression of low or high Candida albicans abundance versus no C. albicans with poor taste, smell, appetite, and undernutrition. Shown are odds ratio (OR), 95\% confidence interval (95\%-CI), and uncorrected $p$ values. Asterisks indicate statistical significance $(p<0.008$ for poor taste; $p<0.013$ for poor smell (Bonferroni corrected); or $p<0.05$ for poor appetite or undernutrition). Adjusted models include covariates: age, dentition, sex, educational status, smoking status, medication use, CESD-score, MMSE-score. Poor taste: total taste score $<6$, sweet score $<2$, sour score $<2$, salty score $<2$, bitter score $<1$, umami score $<1$. Poor smell: TDI-score $\leq 19.5$, T-score $\leq 2.5$, D-score $\leq 7$, I-score $\leq 9$. Poor appetite: Council of Nutrition Appetite Questionnaire score $<28$. Undernutrition: $>5 \%$ bodyweight loss averaged over 2 years or $\mathrm{BMI}<20$ (if age $<70$ ) or $\mathrm{BMI}<22$ (if age $>70$ ) or both.

The association of oral microbiota with taste function has never been studied in a cohort as large as ours, nor specifically in older adults ${ }^{8-11}$. Contrary to poor smell, but consistent with previous studies ${ }^{8,10}$, neither alphadiversity, nor beta-diversity were associated with poor taste. Cattaneo et al. ${ }^{10}$ attributed the lack of associations with alpha- or beta-diversity in part to the limited inter-personal variation in oral microbiota compared to other body sites. However, in our sample of older adults we did find differences in both alpha- and beta- diversity for outcomes other than poor taste. As did the previous studies ${ }^{8-11}$, we identified several differentially abundant taxa for taste in our univariate DESeq analysis. However, unlike these studies, we adjusted our analysis for age and dentition. This rendered the associations with individual taxa non-significant. It is possible that several of the associations found in the previous studies would disappear when adjusting for age or oral health status.

Associations between the oral microbiota and poor oral health ${ }^{24}$, and between poor oral health and poor appetite $^{6}$ have been established in literature. However, the direct association of the oral microbiota with poor 
appetite has not been studied before. In our sample, lower alpha-diversity was associated with poor appetite. Poor appetite also explained a significant amount of variance in beta-diversity and was significantly associated with the microbiota as confirmed by our CCA analysis. Moreover, poor appetite was associated with a significant $\log 2$ fold change in numerous bacterial taxa, but these associations did not hold after adjustment for age and dentition. This suggests that both the oral microbiota and appetite are associated with older age and/or dentition. Since we did find several significant associations before adjustment of the analysis, an indirect relation of oral microbiota with appetite confounded by oral health (or edentation) is possible, but a direct association is less likely.

Although we found associations of alpha- and beta-diversity with poor smell and poor appetite, we found no associations with undernutrition, nor with BMI, previous weight change, FFMI, or ASMM. This could be due to the relatively vital, community-dwelling older population we studied. Undernutrition in community-dwelling older adults is typically less prevalent or pronounced than in less resilient and institutionalized older adults ${ }^{29}$. Moreover, undernutrition is a complex, multi-factorial condition ${ }^{6}$ that may only be expressed when sufficient contributing factors are present. Even if exposed to less desirable, so-called "dysbiotic" oral microbiota, our population may be resilient from developing undernutrition due to sufficient protective circumstances. Possibly, only when enough factors predispose an individual to undernutrition, does the oral microbiota play an active contributing role in its etiology. This would result in a notion of opportunistic dysbiotic microbiota, similar to the way in which an opportunistic bacterium only causes disease under specific circumstances. Future studies, in more sub-populations, are needed to identify what characterizes dysbiotic oral microbiota in regard to undernutrition.

In addition to the tongue microbiota, we explored the relation of C. albicans with poor taste, smell, appetite, and undernutrition. Candida and candidiasis have previously been suggested to affect both undernutrition ${ }^{7,15}$ and taste function ${ }^{16}$. Contrary to the study by Sakashita et al. ${ }^{16}$, high C. albicans abundance was not associated with poor taste in our population. The discrepancy between studies may be due to differences in analysis techniques or study population. Whereas we determined C. albicans abundance by qPCR and studied older adults, Sakashita, et al. determined C. albicans presence by microscopic examination of cultured tongue scrapings in young adults. To our knowledge we are the first to evaluate the association of oral Candida with poor smell. In our exploratory study we found some evidence that high C. albicans abundance is associated with poor discriminatory smell function (borderline significant). However, we found no associations with the total, threshold, or identification smell scores. Possibly the association of $C$. albicans with a diminished ability to discriminate between odors is due to higher levels of inflammation in the nasopharynx. More dedicated research is needed to confirm the relationship between C. albicans and smell function.

Some strengths and limitations to this study are worth mentioning. First, in comparison to previous studies we included a large population ${ }^{8-11}$. We also evaluated the full spectrum of the tongue microbiota using $16 \mathrm{~S}$ rRNA sequencing, rather than depending on targeted culture-dependent techniques 9 . Third, we corrected our final DESeq analysis for multiple testing and adjusted for possible confounding by age and dentition, which appear to be the most important microbiota determinants based on our results. However, the possibility of residual confounding remains. Finally, we considered associations with both overall community structure (alpha- and beta-diversity measures) and individual species. A potential limitation of this study is its cross-sectional design, which keeps us from evaluating causality. Due to complex host-microbe interactions bi-directionality of many associations seems likely. However, as little to no research has yet been done on the topic, establishing cross-sectional associations serves as an important first explorative step. We did not conduct full metagenomics, nor could we assess the functional capacity of the oral microbiome. Possibly, there was no association with the microbial community in some instances, but there would be with bacterial metabolic pathways. Finally, we assessed the oral microbiota on the posterior tongue dorsum, which is only one of many bacterial niches in the oral cavity ${ }^{22}$, although we believe it to be the most suitable for our study as the tongue dorsum is a major microbial reservoir due to its papillary structure with bacterial loads increasing from anterior to posterior ${ }^{8}$. Additionally, the tongue microbiota are most approximate to the taste receptors.

In conclusion, we found several associations of the tongue microbiota with poor smell and poor appetite, but not with poor taste or undernutrition. Specifically, a lower abundance of $S$. salivarius was associated with poor smell, even after adjustment for age or dentition. These results need to be further explored in future studies, but do suggest a host-microbe interaction with regard to smell function and appetite.

\section{Methods}

Participants. This concerns a cross-sectional ancillary-study of the ongoing LASA-study ${ }^{17-19,30}$. LASA participants with available body measurements from the 2015/16 LASA data-collection wave $(n=1642)$ were prescreened for inclusion based on LASA data. Eligible participants $(n=727)$ were then contacted by phone and screened for the remaining exclusion criteria. Exclusion criteria were: age $<65$ years, active institutionalization or $>4$ weeks institutionalization in the three months prior to this study, over-nutrition established either by $\mathrm{BMI}>30 \mathrm{~kg} / \mathrm{m}^{2}$ on last LASA measurement $(2015 / 16)$ or $>2 \%$ body weight gain between latest two LASA measurements (2011/13 to 2015/16), diagnosed active malignancy, systemic antibiotics, immune suppression, and MMSE-score $<18$. Informed consent and all current measurements were obtained during a single home visit $(\mathrm{n}=360)$, in 2017/18. In 1 participant no accurate body measurements could be obtained and in 3 participants no tongue swab could be obtained, leaving 356 participants for the statistical analyses. This study was approved by the local medical ethics committee (Medisch Ethische Toetsingscommissie VUmc, The Netherlands) and carried out in accordance with the Declaration of Helsinki.

Anthropometrics. Participants wore only undergarments when measuring body weight. Some wore indoors clothing without shoes, in which case $1 \mathrm{~kg}$ was subtracted ${ }^{31}$. Weight change was calculated in $\%$ bodyweight averaged over the last 2 years. Undernutrition was defined either by low BMI (BMI $<20 \mathrm{~kg} / \mathrm{m}^{2}$ if age $<70$ years or 
$\mathrm{BMI}<22 \mathrm{~kg} / \mathrm{m}^{2}$ if age $\geq 70$ years $)^{20}$, or by more than $5 \%$ bodyweight loss over 2 years. Body impedance analysis was performed using the BodyStat 1500MDD device (Bodystat Ltd., Isle of Man, UK). FFMI and ASMM were predicted using the validated formulas from Kyle et al. ${ }^{32}$ and Sergi et al. ${ }^{33}$, respectively ${ }^{34}$. Blood Pressure was measured at the left arm in duplicate using an automatic Omron M7 device (Omron Corporation, Tokyo, Japan).

Appetite, oral health, food intake, and covariates. Poor appetite was defined as CNAQ-score $<28^{21}$. Oral health was evaluated using a questionnaire previously used in LASA ${ }^{35}$. Dentition was categorized as follows: no original teeth left ("no teeth"), $\leq 7$ teeth in either upper or lower jaw left ("some teeth), or $>7$ teeth in both the upper and lower jaw ("most-all teeth"). The 238-item Dutch version of the Food Frequency Questionnaire (FFQ) from the Healthy Life in an Urban Setting study was used to assess energy intake (kcal/d) and macronutrient intake $(\mathrm{EN} \%)^{36}$. This FFQ was validated in a cohort of Dutch older adults ${ }^{37}$. Data on socio-demographics (age, sex, educational status), lifestyle factors (Alcohol use ${ }^{38}$ and smoking status), co-morbidities (CES-D depression scores $^{39}$, medication use), and cognitive status (MMSE-score ${ }^{40}$ ) were previously collected by LASA. Education was categorized as low (elementary education or lower), moderate (lower vocational education through general secondary education), and high (higher vocational education through university education). Alcohol use was categorized as none, light, moderate, or excessive based on the Garretsen alcohol consumption index ${ }^{38}$. Medication use was categorized as no medication, 1-4 medicines, $>4$ medicines.

Sensory testing. Taste function was assessed using commercially available taste strips (Burghart Messtechnik GmbH, Wedel, Germany). The test consisted of 22 paper taste strips, 20 of which are impregnated with increasing concentrations of sweet, sour, salty, bitter, and umami tastes, 2 are tasteless ${ }^{41}$, reproducing the test validated by Mueller et al. ${ }^{42}$. Each individual taste score ranges from 0 to 4 , and the total score ranges from 0 to 20. A total taste score $<6$, sweet score $<2$, sour score $<2$, salty score $<2$, bitter score $<1$, or umami score $<1$ was considered poor.

Smell function was measured with the commercially available Sniffin' Sticks (Burghart Messtechnik GmbH, Wedel, Germany), consisting of an odor identification test (score 0-16), an odor discrimination test (score 0-16), and an odor threshold test (score 0-16), which sum up to form the total Threshold-Discrimination-Identification (TDI-) score ${ }^{43}$. An identification score $\leq 9$, discrimination score $\leq 7$, threshold score $\leq 2.5$, or TDI-score $\leq 19.5$ was considered poor ${ }^{43}$. Not all participants completed the threshold and discrimination tests, as they are time consuming, can be considered too burdensome, and not all researchers performing the home visits were equipped to carry them out. The smell identification test was performed in all visits and was therefore used as our overall measurement for olfactory function ${ }^{44-49}$. Whether the discrimination and threshold tests were performed was decided prior to the visit based on the participant's preference and researcher available. Participants were asked not to smoke, chew gum, eat, or drink anything other than water in the hour preceding the sensory tests.

Tongue swap. Participants were asked to stay fasted and not smoke or chew gum in the hour prior to tongue sampling, nor did they brush their teeth that day. Tongue swabs were collected in duplicate by stroking a Copan eNAT swab (Copan Italia S.p.A., Bréscia, Italy) over the posterior tongue dorsum for four times. The swab was then inserted in a tube containing the eNAT RNA/DNA stabilizing and preservation medium. It was chilled in an icepack until storage at $-80^{\circ} \mathrm{C}$ on the same day.

16S rRNA analysis. 16S rDNA amplicon sequencing of the V4 hypervariable region was performed as described previously ${ }^{50}$. Each sample was PCR amplified using 1 ng of template DNA with primers F515/R806, targeting the V4 hypervariable region of the $16 \mathrm{~s}$ ribosomal gene ${ }^{51}$. In each batch of samples, a mixed pure culture isolates (mock), blanco extraction controls, pooled salivary extraction controls and pooled DNA amplification controls were included. The amount of DNA per sample was quantified using the Quant-iT ${ }^{\mathrm{ma}}$ PicoGreen $^{\circ}$ dsDNA Assay Kit (Thermo Fisher Scientific). The amplicon libraries were pooled in equimolar amounts and purified using the IllustraTM GFXTM PCR DNA and Gel Band Purification Kit (GE Healthcare, Eindhoven, The Netherlands). Amplicon quality and size was analyzed on the Fragment Analyzer (Advanced Analytical). Paired-end sequencing of amplicons was conducted in five separate runs on the Illumina MiSeq platform (Illumina, Eindhoven, The Netherlands). Denoising of sequence data and identification of ASVs were performed using the DADA2 (1.12.1) pipeline ${ }^{52}$.

Candida qPCR. Quantitative assessment of C. albicans abundance was performed by quantitative PCR using primers listed in Supplementary Table S7 ${ }^{53}$. qPCR was performed in RT PCR master mix (Diagenode, Seraing, B) on an Applied Biosystems 7500 RT PCR system during 40 cycles of with a denaturation step at $95.0^{\circ} \mathrm{C} \mathrm{for} 15 \mathrm{~s}$ and an annealing/elongation step at $60.0^{\circ} \mathrm{C}$ for $1 \mathrm{~min}$. A standard curve was established by analysis of genomic DNA of C. albicans ATCC 90,028. Cell densities were estimated by accounting for the elution volume used after DNA extraction $(60 \mu \mathrm{l})$ and assuming a (diploid) genome size of $14 \mathrm{Mb}$ for C. albicans. C. albicans abundance was then categorized into 3 groups: no C. albicans present, low C. albicans abundance ( 0 counts through the median number of counts), and high C. albicans abundance (the median number of counts through the highest number of counts).

Statistical analysis. All statistical analyses of the microbiota were performed using R version 3.5.1 ${ }^{54}$. All figures were composed using the ggplot2 package version 3.2.15. Multivariate analyses and ordinations were performed using the vegan package, version $2.5-3^{56}$. This package was also used to calculate the inverse Simpson and Shannon $\alpha$-diversities. Associations of the clinical variables with the $\alpha$-diversity measures were determined 
using univariate linear regression from the base $\mathrm{R}$ package. For all analyses, with the exception of those concerning a-diversity, the $16 \mathrm{~S}$ data was filtered to include only those ASVs that contribute to the first $97.5 \%$ of all counts in the data. The $\mathrm{R}^{2}$ in Bray-Curtis $\beta$-diversity for the clinical variables was determined by fitting vectors for continuous variables and weighted centroids for categorical variables. Significance of these $\mathrm{R}^{2}$ values was calculated using PERMANOVA analysis. The metric multi-dimensional scaling ordination is a distance-based method; in this case Bray-Curtis distances were used. This is a non-Euclidean metric, therefore the reported explained variance for the first two axes are based on Cailliez-corrected eigenvalues ${ }^{57}$. Canonical correspondence analysis (CCA) is a method often used to identify correlations between two data matrixes, in this case between the microbiota data and taste, smell, appetite, and undernutrition. The multivariate models fitted by CCA were tested by permutation analysis in order to produce Type III (marginal) $p$ values for the terms included in the model. $10 \times 10^{2}$ permutations were used for all reported results. The species weights for this model are reported as weighted averages calculated from the microbiota composition and the model variables. Regression on the microbiota count data was in all cases performed using the DESeq2 package, version $1.20 .0^{58}$. Log2 fold change in microbiota count was calculated for age, dentition, poor smell, poor taste, poor appetite, and undernutrition univariately and adjusted for age, dentition, or age and dentition. $p$ values were corrected for multiple testing according to the Benjamini-Hochberg procedure.

For the statistical analyses concerning Candida data, SPSS software version 22 (SPSS Inc., Chicago Illinois) was used. The associations of $C$. albicans (low abundance versus none, and high abundance versus none) with all taste and smell scores, poor appetite, and undernutrition were explored with logistic regression. These models were first run univariately and then adjusted for age, dentition, sex, educational status, smoking status, medication use, CESD-score, MMSE-score. As all individual taste scores make up the overall taste test and all individual smell scores make up the overall smell test, Bonferroni correction for multiple testing was carried out for models with taste or smell scores as outcome. Bonferroni corrected alpha-levels used for statistical significance were 0.008 $(=0.05 / 6)$ for taste and $0.013(=0.05 / 4)$ for smell, respectively. Bonferroni uncorrected $p$ values are reported.

\section{Data availability}

The 16S rRNA gene sequences that support the findings of this study have deposited in the restricted access data repository: the European Genome-Phenome Archive (https://ega-archive.org/), dataset ID: EGAD00001007705. The 16S rRNA gene sequences, as well as the associated metadata are available upon request from the Longitudinal Aging Study Amsterdam (https://lasa-vu.nl). The data are not publicly available due to ethical and privacy considerations.

Received: 12 July 2021; Accepted: 12 November 2021

Published online: 01 December 2021

\section{References}

1. United Nations, Department of Economic and Social Affairs, Population Division (2019). World Population Prospects 2019: Highlights (ST/ESA/SER.A/423).

2. Leij-Halfwerk, S. et al. Prevalence of protein-energy malnutrition risk in European older adults in community, residential and hospital settings, according to 22 malnutrition screening tools validated for use in adults $>/=65$ years: A systematic review and meta-analysis. Maturitas 126, 80-89 (2019).

3. Keller, H. H., Ostbye, T. \& Goy, R. Nutritional risk predicts quality of life in elderly community-living Canadians. J. Gerontol. A Biol. Sci. Med. Sci. 59(1), 68-74 (2004).

4. Correia, M. I. \& Waitzberg, D. L. The impact of malnutrition on morbidity, mortality, length of hospital stay and costs evaluated through a multivariate model analysis. Clin. Nutr. 22(3), 235-239 (2003).

5. van der Pols-Vijlbrief, R., Wijnhoven, H. A., Schaap, L. A., Terwee, C. B. \& Visser, M. Determinants of protein-energy malnutrition in community-dwelling older adults: A systematic review of observational studies. Ageing Res Rev. 18, 112-131 (2014).

6. Wysokinski, A., Sobow, T., Kloszewska, I. \& Kostka, T. Mechanisms of the anorexia of aging-A review. Age (Dordr). 37(4), 9821 (2015).

7. Poisson, P., Laffond, T., Campos, S., Dupuis, V. \& Bourdel-Marchasson, I. Relationships between oral health, dysphagia and undernutrition in hospitalised elderly patients. Gerodontology 33(2), 161-168 (2016).

8. Besnard, P. et al. Obese subjects with specific gustatory papillae microbiota and salivary cues display an impairment to sense lipids. Sci. Rep. 8(1), $6742(2018)$.

9. Feng, Y. et al. The associations between biochemical and microbiological variables and taste differ in whole saliva and in the film lining the tongue. Biomed. Res. Int. 2018, 2838052. https://doi.org/10.1155/2018/2838052 (2018).

10. Cattaneo, C. et al. New insights into the relationship between taste perception and oral microbiota composition. Sci. Rep. 9(1), 3549 (2019).

11. Cattaneo, C., Riso, P., Laureati, M., Gargari, G. \& Pagliarini, E. Exploring associations between interindividual differences in taste perception, oral microbiota composition, and reported food intake. Nutrients 11(5), 1167 (2019).

12. Human Microbiome Project Consortium. Structure, function and diversity of the healthy human microbiome. Nature. 486(7402), 207-214 (2012)

13. Neyraud, E. \& Morzel, M. Biological films adhering to the oral soft tissues: Structure, composition, and potential impact on taste perception. J. Texture Stud. 50(1), 19-26 (2019).

14. Francois, A. et al. Olfactory epithelium changes in germfree mice. Sci. Rep. 6, 24687 (2016).

15. Paillaud, E. et al. Oral candidiasis and nutritional deficiencies in elderly hospitalised patients. Br. J. Nutr. 92(5), 861-867 (2004).

16. Sakashita, S., Takayama, K., Nishioka, K. \& Katoh, T. Taste disorders in healthy "carriers" and "non-carriers" of Candida albicans and in patients with candidosis of the tongue. J. Dermatol. 31(11), 890-897 (2004).

17. Hoogendijk, E. O. et al. The Longitudinal Aging Study Amsterdam: Cohort update 2016 and major findings. Eur. J. Epidemiol. 31(9), 927-945 (2016).

18. Hoogendijk, E. O. et al. The Longitudinal Aging Study Amsterdam: Cohort update 2019 and additional data collections. Eur. J. Epidemiol. 35(1), 61-74 (2020).

19. Huisman, M. et al. Cohort profile: The Longitudinal Aging Study Amsterdam. Int. J. Epidemiol. 40(4), 868-876 (2011).

20. Cederholm, T. et al. Diagnostic criteria for malnutrition-An ESPEN consensus statement. Clin. Nutr. 34(3), 335-340 (2015). 
21. Hanisah, R., Suzana, S. \& Lee, F. S. Validation of screening tools to assess appetite among geriatric patients. J. Nutr. Health Aging. 16(7), 660-665 (2012).

22. Dewhirst, F. E. et al. The human oral microbiome. J. Bacteriol. 192(19), 5002-5017 (2010).

23. Kaci, G. et al. Anti-inflammatory properties of Streptococcus salivarius, a commensal bacterium of the oral cavity and digestive tract. Appl. Environ. Microbiol. 80(3), 928-934 (2014).

24. Asakawa, M. et al. Tongue microbiota and oral health status in community-dwelling elderly adults. $m S p h e r e$ 3(4), e00332-18 (2018).

25. Zupancic, K., Kriksic, V., Kovacevic, I. \& Kovacevic, D. Influence of oral probiotic Streptococcus salivarius K12 on ear and oral cavity health in humans: Systematic review. Probiotics Antimicrob. Proteins 9(2), 102-110 (2017).

26. Tagg, J. R. \& Dierksen, K. P. Bacterial replacement therapy: Adapting 'germ warfare' to infection prevention. Trends Biotechnol. 21(5), 217-223 (2003).

27. Guglielmetti, S. et al. Oral bacteria as potential probiotics for the pharyngeal mucosa. Appl. Environ. Microbiol. 76(12), 3948-3958 (2010).

28. Huttenbrink, K.B., Hummel, T., Berg, D., Gasser, T. \& Hahner, A. Olfactory dysfunction: common in later life and early warning of neurodegenerative disease. Dtsch Arztebl Int. 110(1-2), 1-7, e1 (2013).

29. Pavlovic, J., Racic, M., Ivkovic, N. \& Jatic, Z. Comparison of nutritional status between nursing home residents and community dwelling older adults: A cross-sectional study from bosnia and herzegovina. Mater. Sociomed. 31(1), 19-24 (2019).

30. Fluitman, K. S. et al. Poor taste and smell are associated with poor appetite, macronutrient intake, and dietary quality but not with undernutrition in older adults. J. Nutr. 151(3), 605-614 (2021).

31. Whigham, L. D., Schoeller, D. A., Johnson, L. K. \& Atkinson, R. L. Effect of clothing weight on body weight. Int. J. Obes. (Lond.) 37(1), 160-161 (2013).

32. Kyle, U. G., Genton, L., Karsegard, L., Slosman, D. O. \& Pichard, C. Single prediction equation for bioelectrical impedance analysis in adults aged 20-94 years. Nutrition 17(3), 248-253 (2001).

33. Sergi, G. et al. Assessing appendicular skeletal muscle mass with bioelectrical impedance analysis in free-living Caucasian older adults. Clin. Nutr. 34(4), 667-673 (2015).

34. Cruz-Jentoft, A. J. et al. Sarcopenia: Revised European consensus on definition and diagnosis. Age Ageing 48(1), 16-31 (2019).

35. Kiesswetter, E., Hengeveld, L. M., Keijser, B. J., Volkert, D. \& Visser, M. Oral health determinants of incident malnutrition in community-dwelling older adults. J. Dent. 85, 73-80 (2019).

36. Beukers, M. H. et al. Development of the HELIUS food frequency questionnaires: ethnic-specific questionnaires to assess the diet of a multiethnic population in The Netherlands. Eur. J. Clin. Nutr. 69(5), 579-584 (2015).

37. Visser, M., Elstgeest, L. E. M., Winkens, L. H. H., Brouwer, I. A. \& Nicolaou, M. Relative validity of the HELIUS Food Frequency Questionnaire for measuring dietary intake in older adult participants of the Longitudinal Aging Study Amsterdam. Nutrients 12(7), 1998 (2020).

38. Garretsen, H. R. F. \& Knibbe, R. A. Alcohol prevalentie onderzoek Rotterdam/Limburg, Landelijk Eindrapport, Ministerie van Welzijn, Volksgezondheid en Cultuur, Leidschendam (1983).

39. Radloff, L. The CES-D scale: A self-reported depression scale for research in the general population. Appl. Psychol. Meas. 1, 385-401 (1977).

40. Folstein, M. F., Folstein, S. E. \& McHugh, P. R. "Mini-mental state" A practical method for grading the cognitive state of patients for the clinician. J. Psychiatr. Res. 12(3), 189-198 (1975)

41. Landis, B. N. et al. "Taste Strips"-a rapid, lateralized, gustatory bedside identification test based on impregnated filter papers. J. Neurol. 256(2), 242-248 (2009).

42. Mueller, C. A., Pintscher, K. \& Renner, B. Clinical test of gustatory function including umami taste. Ann. Otol. Rhinol. Laryngol. 120(6), 358-362 (2011).

43. Hummel, T., Kobal, G., Gudziol, H. \& Mackay-Sim, A. Normative data for the "Sniffin' Sticks" including tests of odor identification, odor discrimination, and olfactory thresholds: An upgrade based on a group of more than 3,000 subjects. Eur. Arch. Otorhinolaryngol. 264(3), 237-243 (2007).

44. Arganini, C. \& Sinesio, F. Chemosensory impairment does not diminish eating pleasure and appetite in independently living older adults. Maturitas 82(2), 241-244 (2015).

45. de Jong, N., Mulder, I., de Graaf, C. \& van Staveren, W. A. Impaired sensory functioning in elders: the relation with its potential determinants and nutritional intake. J. Gerontol. A Biol. Sci. Med. Sci. 54(8), B324-B331 (1999).

46. Fluitman, K. S. et al. The association of olfactory function with BMI, appetite, and prospective weight change in Dutch communitydwelling older adults. J. Nutr. Health Aging 23(8), 746-752 (2019).

47. Gopinath, B. et al. Olfactory impairment in older adults is associated with poorer diet quality over 5 years. Eur. J. Nutr. 55(3), 1081-1087 (2016).

48. Karpa, M. J. et al. Prevalence and neurodegenerative or other associations with olfactory impairment in an older community. J. Aging Health. 22(2), 154-168 (2010).

49. Smoliner, C., Fischedick, A., Sieber, C. C. \& Wirth, R. Olfactory function and malnutrition in geriatric patients. J. Gerontol. A Biol. Sci. Med. Sci. 68(12), 1582-1588 (2013).

50. Keijser, B. J. F. et al. Dose-dependent impact of oxytetracycline on the veal calf microbiome and resistome. BMC Genom. 20(1), 65 (2019).

51. Caporaso, J. G. et al. Global patterns of $16 \mathrm{~S}$ rRNA diversity at a depth of millions of sequences per sample. Proc. Natl. Acad. Sci. U. S. A. 108(Suppl 1), 4516-4522 (2011).

52. Callahan, B. J. et al. DADA2: High-resolution sample inference from Illumina amplicon data. Nat. Methods 13(7), 581-583 (2016).

53. Guiver, M., Levi, K. \& Oppenheim, B. A. Rapid identification of candida species by TaqMan PCR. J. Clin. Pathol. 54(5), 362-366 (2001).

54. R Core Team. R: A Language and Environment for Statistical Computing. Vienna, Austria (2018).

55. Wickham, H. Elegant Graphics for Data Analysis (Springer-Verlag, 2016).

56. Oksanen, J., et al. Vegan: Community Ecology Package. R package 2018.

57. Cailliez, F. The analytical solution of the additive constant problem. Psychometrika 48(2), 305-308 (1983).

58. Love, M. I., Wolfgang, H. \& Anders, S. Moderated estimation of fold change and dispersion for RNA-seq data with DESeq2. Genome Biol. 15(12), 550 (2014).

\section{Acknowledgements}

The authors would like to express their gratitude to Hema J. Nadar, Madelief Wijdeveld, Rachel F. Kaihatu, and Anne C. Hesp for assistance in data-collection. 


\section{Author contributions}

K.F., R.G., M.V., M.N., and B.K. were involved in the conceptualization of the work. K.F. was involved in datacollection. T.J.B., K.F., R.G., and B.K. were involved in data-analysis. All authors have critically revised this work and approved the final version for publication.

\section{Funding}

This study was supported by the European Union Horizon 2020 PROMISS Project 'PRevention Of Malnutrition In Senior Subjects in the EU' (Grant Agreement No. 678732). The content only reflects the author's view and the Commission is not responsible for any use that may be made of the information it contains. The Longitudinal Aging Study Amsterdam is supported by a grant from the Netherlands Ministry of Health Welfare and Sports, Directorate of Long-Term Care. The LASA data collection in 2012-2013 was financially supported by the Netherlands Organization for Scientific Research (NWO) in the framework of the project "New Cohorts of young old in the twenty-first century" (File Number 480-10-014).

\section{Competing interests}

The authors declare no competing interests.

\section{Additional information}

Supplementary Information The online version contains supplementary material available at https:/doi.org/ 10.1038/s41598-021-02558-8.

Correspondence and requests for materials should be addressed to B.J.F.K.

Reprints and permissions information is available at www.nature.com/reprints.

Publisher's note Springer Nature remains neutral with regard to jurisdictional claims in published maps and institutional affiliations.

(c) (i) Open Access This article is licensed under a Creative Commons Attribution 4.0 International cc) License, which permits use, sharing, adaptation, distribution and reproduction in any medium or format, as long as you give appropriate credit to the original author(s) and the source, provide a link to the Creative Commons licence, and indicate if changes were made. The images or other third party material in this article are included in the article's Creative Commons licence, unless indicated otherwise in a credit line to the material. If material is not included in the article's Creative Commons licence and your intended use is not permitted by statutory regulation or exceeds the permitted use, you will need to obtain permission directly from the copyright holder. To view a copy of this licence, visit http://creativecommons.org/licenses/by/4.0/.

(C) The Author(s) 2021 